\title{
The Shapley valuation function for strategic games in which players cooperate*
}

\author{
Luisa Carpente ${ }^{1}$ \\ Balbina Casas-Méndez ${ }^{2}$ \\ Ignacio García-Jurado ${ }^{2} \quad$ Anne van den Nouweland ${ }^{3}$
}

February 19, 2004

\begin{abstract}
In this note we use the Shapley value to define a valuation function. A valuation function associates with every non-empty coalition of players in a strategic game a vector of payoffs for the members of the coalition that provides these players' valuations of cooperating in the coalition. The Shapley valuation function is defined using the lowervalue based method to associate coalitional games with strategic games that was introduced in Carpente et al. (2003). We discuss axiomatic characterizations of the Shapley valuation function.
\end{abstract}

\section{Introduction}

In this note we consider valuation functions. A valuation function associates with every non-empty coalition of players in a strategic game a vector of payoffs for the members of the coalition that provides these players' valuations of cooperating in the coalition. We formulate axioms for such a

\footnotetext{
*The authors acknowledge the financial support of Ministerio de Ciencia y Tecnología, FEDER and Xunta de Galicia through projects BEC2002-04102-C02-02 and PGIDIT03PXIC20701PN.

${ }^{1}$ Departamento de Matemáticas. Facultade de Informática. Universidade da Coruña. 15071 A Coruña, Spain.

${ }^{2}$ Departamento de Estatística e IO. Facultade de Matemáticas. Universidade de Santiago de Compostela. 15782 Santiago de Compostela, Spain.

${ }^{3}$ Corresponding author. Department of Economics, 435 PLC, University of Oregon, Eugene, OR 97403-1285, USA. Email: annev@uoregon.edu
} 
valuation function and prove that there exists a unique valuation function that satisfies these axioms. This valuation function is found by applying Shapley values (cf. Shapley (1953)) to coalitional games that are obtained by applying the lower-value based method to associate a coalitional game with every strategic game. The lower-value based method was introduced in Carpente et al. (2003).

Von Neumann and Morgenstern (1944) explicitly consider the possibility that coalitions of players cooperate in strategic games. To formalize this, they associate with every strategic game a coalitional game in which the worth of a coalition of players represents the worth that these players can jointly obtain when they coordinate their actions. This worth is defined to be the value of the mixed extension of the zero-sum game that the coalition plays with the complementary coalition consisting of all other players. Carpente et al. (2003) axiomatically characterize the method of associating a coalitional game with every strategic game that was proposed by Von Neumann and Morgenstern. In addition, they formulate and axiomatically characterize a variation of Von Neumann and Morgenstern's method by considering lower values of the zero-sum games between coalitions and their complementary coalitions. They argue that the method based on lower values rather than values is more appropriate in situations where it is not reasonable to assume that coalitions of players can mix coordinated actions.

We introduce our framework and axioms in Section 2, in which we also prove that the so-called Shapley valuation function, which is based on the Carpente et al. (2003) method to associate coalitional games with strategic games, is the unique valuation function satisfying all the axioms. We conclude in Section 3 by pointing out how the axioms need to be adapted to axiomatically characterize a valuation function based on Shapley values and the Von Neumann and Morgenstern (1944) method to associate coalitional games with strategic games. In this section, we also point out how one of the axioms, which deals with monotonicity in payoffs, can be replaced by two other axioms that deal with monotonicity when deleting actions. 


\section{A valuation function for strategic games in which players cooperate}

A strategic game $g=\left(N,\left\{X_{i}\right\}_{i \in N},\left\{u_{i}\right\}_{i \in N}\right)$ consists of a set of players $N=$ $\{1, \cdots, n\}$ and, for every player $i \in N$, a set of actions $X_{i}$ available to this player, as well as a payoff function $u_{i}: \prod_{j \in N} X_{j} \rightarrow \mathbb{R}$. In this note, we consider only finite strategic games, i.e. games with finitely many players in which the action set $X_{i}$ is finite for each player $i$. The class of finite strategic games with player set $N$ is denoted by $G^{N}$.

We assume that in a strategic game $g \in G^{N}$ a coalition of players $S \subset N$ is able to coordinate and play any action profile $x_{S} \in X_{S}:=\prod_{i \in S} X_{i}$. We associate with each game $g \in G^{N}$ and coalition $S \subset N$ the payoffs attainable in game $g$ by the players in $S$ if they decide to cooperate, independent of which actions are played by the other players. A valuation function is a map $\varphi$ that associates a payoff vector $\varphi(S, g) \in \mathbb{R}^{S}$ with every game $g \in G^{N}$ and non-empty coalition $S \subset N$, where $\varphi_{i}(S, g)$ provides a valuation for player $i$ of cooperating in coalition $S$ in game $g$, for each $i \in S$.

We consider the following properties for a valuation function. Most of these properties are inspired by analogous ones introduced in Carpente et al. (2003).

Individual objectivity states that if a player gets the same payoff for any possible action tuple in a game, then the valuation for this player of forming a singleton coalition is equal to this amount.

Individual objectivity. For all $g \in G^{N}$ and all players $i \in N$, if $c \in \mathbb{R}$ is such that $u_{i}(x)=c$ for all $x \in X_{N}$, then

$$
\varphi_{i}(\{i\}, g)=c
$$

Monotonicity states that the valuation for a player of forming a singleton coalition does not decrease if his payoff in the strategic game weakly increases for all possible action tuples. 
Monotonicity. For all strategic games $g=\left(N,\left\{X_{i}\right\}_{i \in N},\left\{u_{i}\right\}_{i \in N}\right)$ and $g^{\prime}=\left(N,\left\{X_{i}\right\}_{i \in N},\left\{u_{i}^{\prime}\right\}_{i \in N}\right)$, and player $i \in N$ such that $u_{i}(x) \geq$ $u_{i}^{\prime}(x)$ for all $x \in X_{N}$,

$$
\varphi_{i}(\{i\}, g) \geq \varphi_{i}\left(\{i\}, g^{\prime}\right)
$$

Irrelevance of strongly dominated actions states that the valuation for a player of forming a singleton coalition does not change if in the strategic game he loses the ability to use an action that is weakly worse for him than another of his actions, no matter what actions the other players choose. To understand the relevance of this property (as well as of the next one, irrelevance of weakly dominated threats), note that the valuation for a player of forming a singleton coalition is interpreted as the payoff that this player can guarantee himself independent of what actions are played by the other players.

Irrelevance of strongly dominated actions. In a game $g \in G^{N}$, an action $x_{i} \in X_{i}$ of player $i \in N$ is strongly dominated if there exists an action $x_{i}^{\prime} \in X_{i}, x_{i}^{\prime} \neq x_{i}$, such that $u_{i}\left(x_{i}^{\prime}, x_{N \backslash i}\right) \geq u_{i}\left(x_{i}, x_{N \backslash i}\right)$ for all $x_{N \backslash i} \in X_{N \backslash i}$. For all $g \in G^{N}$ and $i \in N$, if action $x_{i} \in X_{i}$ is strongly dominated, then $\varphi_{i}(\{i\}, g)=\varphi_{i}\left(\{i\}, g^{\prime}\right)$, where $g^{\prime} \in G^{N}$ is the game obtained from $g$ by deleting action $x_{i}$.

Irrelevance of weakly dominated threats states that the valuation for a player $i$ of forming a singleton coalition is not affected if another player $j$ is prohibited from using an action whose deletion does not change player $i$ 's worst-case scenario.

Irrelevance of weakly dominated threats. In a game $g \in G^{N}$, an action $x_{j} \in X_{j}$ of a player $j \in N$ is a weakly dominated threat to player $i \in N, i \neq j$, if for every $x_{N \backslash j} \in X_{N \backslash j}$ there exists an action $x_{j}^{\prime} \in X_{j}$, $x_{j}^{\prime} \neq x_{j}$, such that $u_{i}\left(x_{j}^{\prime}, x_{N \backslash j}\right) \leq u_{i}\left(x_{j}, x_{N \backslash j}\right)$. For all $g \in G^{N}$ and players $i, j \in N, i \neq j$, if action $x_{j} \in X_{j}$ is a weakly dominated threat to player $i$, then $\varphi_{i}(\{i\}, g)=\varphi_{i}\left(\{i\}, g^{\prime}\right)$, where $g^{\prime} \in G^{N}$ is the game obtained from $g$ by deleting action $x_{j}$. 
We now introduce some additional notation in order to be able to formulate the next property. Let $g=\left(N,\left\{X_{i}\right\}_{i \in N},\left\{u_{i}\right\}_{i \in N}\right) \in G^{N}$ and $S \subset N$, $S \neq \emptyset$. Suppose that the members of coalition $S$ decide to merge and act as one player. In order to study the opportunities of $S$ as a coalition, we introduce a new player $i(S)$ with action set $X_{i(S)}:=X_{S}$ and utility function $u_{i(S)}: \Pi_{j \in(N \backslash S) \cup\{i(S)\}} X_{j} \rightarrow \mathbb{R}$ defined by $u_{i(S)}\left(x_{N \backslash S}, x_{i(S)}\right)=$ $\sum_{j \in S} u_{j}\left(x_{N \backslash S}, x_{S}\right)$ for all $x_{N \backslash S} \in X_{N \backslash S}$ and all $x_{i(S)}=x_{S} \in X_{S}=X_{i(S)}$. Denote $N(S):=(N \backslash S) \cup\{i(S)\}$. The strategic game $g(S) \in G^{N(S)}$ is defined by $g(S)=\left(N(S),\left\{X_{i}\right\}_{i \in N(S)},\left\{u_{i}\right\}_{i \in N(S)}\right)$. The property merge invariance states that the total valuation for the players in $S$ of forming coalition $S$ in the game $g$ is the same as the valuation for player $i(S)$ of forming a singleton coalition in the game $g(S)$. The interpretation of this property is that a coalition of players cannot influence their joint valuation by merging and acting as one player. Such a requirement seems natural as a coalition of players who decide to cooperate is supposed to act in the best interest of the group.

Merge invariance. For all $g \in G^{N}$ and non-empty $S \subset N$,

$$
\sum_{i \in S} \varphi_{i}(S, g)=\varphi_{i(S)}(\{i(S)\}, g(S))
$$

In the literature on the Shapley value and other solution concepts for coalitional games, a principle of reciprocity between the players is often used. We use balanced contributions, as introduced in Myerson (1980). The principle of balanced contributions asserts that for any two players the gains or losses that they can inflict on each other by leaving the game should be equal. Myerson used this principle to extend the Shapley value to a setting of coalitional games without transferable utility with conferences structures.

Our aim is to apply the principle of balanced contributions to valuation functions. In this setting, rather than considering that players leave the game, we assert that players leave the coalition of cooperating players and consider the losses or gains that this inflicts on other players in the cooperating coalition. 
Balanced contributions. For all $g \in G^{N}$ and non-empty $S \subset N$, and all $i, j \in S$,

$$
\varphi_{i}(S, g)-\varphi_{i}(S \backslash\{j\}, g)=\varphi_{j}(S, g)-\varphi_{j}(S \backslash\{i\}, g) .
$$

As we pointed out above, in coalitional games the property balanced contributions is intimately connected with the Shapley value. This is still true in the context of valuation functions, in which we associate payoff vectors with coalitions of cooperating players in strategic games. We show in Theorem 1 below that there exists a unique valuation function that satisfies the properties that we have stated above. This valuation function is derived from the Shapley value in the following manner.

Take a strategic game $g \in G^{N}$. With this game we associate the coalitional game $\left(N, \underline{v}_{g}\right)$ as defined in Carpente et al. (2003). A coalitional game is a pair $(N, v)$ consisting of a player set $N=\{1, \ldots, n\}$ and a characteristic function $v: 2^{N} \rightarrow \mathbb{R}$ that assigns to each coalition $S \subset N$ its worth $v(S)$ representing the benefits that this coalition can guarantee its members independently of what the other players (those in $N \backslash S$ ) do (by convention, $v(\emptyset)=0)$. In the game $\left(N, \underline{v}_{g}\right)$, the worth $\underline{v}_{g}(S)$ of a coalition of players $S \subset N$ is the lower value of a finite two-person zero-sum game between coalition $S$ on the one hand and the coalition $N \backslash S$ on the other hand. For any non-empty coalition $S \subset N, S \neq N$, the two-player zero-sum game $g_{S}$ is defined by

$$
g_{S}=\left(\{S, N \backslash S\},\left\{X_{S}, X_{N \backslash S}\right\},\left\{u_{S},-u_{S}\right\}\right),
$$

where, for all $T \subset N, X_{T}=\prod_{i \in T} X_{i}$ and $u_{T}=\sum_{i \in T} u_{i}$. This game has two players, coalitions $S$ and $N \backslash S$. The actions available to each of these two coalitions are all the combinations of the actions available to its members in the game $g$ and for every possible action tuple the payoff to coalition $S$ is the sum of the payoffs of its members while the payoff to coalition $N \backslash S$ is the opposite of this. The lower value of this game is $\underline{v}_{g}(S)=$ $\max _{x_{S} \in X_{S}} \min _{x_{N \backslash S} \in X_{N \backslash S}} u_{S}\left(x_{S}, x_{N \backslash S}\right)$. This is the worth that the players in coalition 
$S$ can secure for themselves by coordinating their actions even if the players in $N \backslash S$ cooperate to keep the worth of coalition $S$ as low as possible. The worth of the grand coalition $N$ is simply defined as $\underline{v}_{g}(N)=\max _{x \in X_{N}} u_{N}(x)$.

The Shapley valuation function $\phi_{\underline{V}}$ assigns to each strategic game $g \in G^{N}$ and non-empty coalition $S \subset N$ the Shapley value $\phi\left(S, \underline{v}_{g}\right)$ of the coalitional game $\left(S, \underline{v}_{g}\right)$ associated with $g$ and $S$, which is defined by

$$
\phi_{i}\left(S, \underline{v}_{g}\right)=\sum_{T \subset S, i \in T} \frac{(|T|-1) !(|S|-|T|) !}{|S| !}\left(\underline{v}_{g}(T)-\underline{v}_{g}(T \backslash\{i\})\right)
$$

for all $i \in S$.

Theorem 1 The Shapley valuation function $\phi_{\underline{V}}$ is the unique valuation function satisfying individual objectivity, monotonicity, irrelevance of strongly dominated actions, irrelevance of weakly dominated threats, merge invariance, and balanced contributions.

Proof. Existence. The proof that $\phi_{\underline{V}}$ satisfies the five properties individual objectivity, monotonicity, irrelevance of strongly dominated actions, irrelevance of weakly dominated threats, and merge invariance, uses the results in Carpente et al. (2003). In that paper it is proved that the lower value method, which associates with each strategic game $g \in G^{N}$ the coalitional game $\left(N, \underline{v}_{g}\right)$, is the unique method of associating a coalitional game with each strategic game that satisfies the appropriate equivalents of these five properties.

To check that $\phi_{\underline{V}}$ satisfies individual objectivity, let $g \in G^{N}, i \in N$, and $c \in \mathbb{R}$ be such that $u_{i}(x)=c$ for all $x \in X_{N}$. Then $\left(\phi_{\underline{V}}\right)_{i}(\{i\}, g)=$ $\phi_{i}\left(\{i\}, \underline{v}_{g}\right)=\underline{v}_{g}(\{i\})=c$, where the first equality simply uses the definition of the Shapley valuation function, the second one follows from efficiency of the Shapley value, and the third one follows from individual objectivity of the lower value method.

To check that $\phi_{\underline{V}}$ satisfies monotonicity, let $g=\left(N,\left\{X_{i}\right\}_{i \in N},\left\{u_{i}\right\}_{i \in N}\right) \in$ $G^{N}, g^{\prime}=\left(N,\left\{X_{i}\right\}_{i \in N},\left\{u_{i}^{\prime}\right\}_{i \in N}\right) \in G^{N}$, and $i \in N$ be such that $u_{i}(x) \geq u_{i}^{\prime}(x)$ for all $x \in X_{N}$. Then $\left(\phi_{\underline{V}}\right)_{i}(\{i\}, g)=\phi_{i}\left(\{i\}, \underline{v}_{g}\right)=\underline{v}_{g}(\{i\}) \geq \underline{v}_{g^{\prime}}(\{i\})=$ 
$\phi_{i}\left(\{i\}, \underline{v}_{g^{\prime}}\right)=\left(\phi_{\underline{V}}\right)_{i}\left(\{i\}, g^{\prime}\right)$, where the inequality follows from monotonicity of the lower value method.

To check that $\phi_{\underline{V}}$ satisfies irrelevance of strongly dominated actions, we use that the equivalent of this property for the lower value method implies that $\underline{v}_{g}(\{i\})=\underline{v}_{g^{\prime}}(\{i\})$ for any two strategic games $g, g^{\prime} \in G^{N}$ where the game $g^{\prime}$ is the game obtained from $g$ by deleting a strongly dominated action $x_{i} \in X_{i}$ of player $i \in N$. Irrelevance of weakly dominated threats of $\phi_{\underline{V}}$ follows in a similar manner from the equivalent property for the lower value method.

To check that $\phi_{\underline{V}}$ satisfies merge invariance, let $g \in G^{N}$ and $S \subset N$, $S \neq \emptyset$. Then $\sum_{i \in S}\left(\underline{\phi}_{\underline{V}}\right)_{i}(S, g)=\sum_{i \in S} \phi_{i}\left(S, \underline{v}_{g}\right)=\underline{v}_{g}(S)=\underline{v}_{g(S)}(i(S))=$ $\phi_{i(S)}\left(\{i(S)\}, \underline{v}_{g(S)}\right)=\left(\phi_{\underline{V}}\right)_{i(S)}(\{i(S)\}, g(S))$, where the second and fourth equalities use efficiency of the Shapley value and the third one follows from merge invariance of the lower value method.

To check that $\phi_{\underline{V}}$ satisfies balanced contributions, let $g \in G^{N}, S \subset N$, and $i, j \in S$. Then $\left(\phi_{\underline{V}}\right)_{i}(S, g)-\left(\phi_{\underline{V}}\right)_{i}(S \backslash\{j\}, g)=\phi_{i}\left(S, \underline{v}_{g}\right)-\phi_{i}\left(S \backslash\{j\}, \underline{v}_{g}\right)=$ $\phi_{j}\left(S, \underline{v}_{g}\right)-\phi_{j}\left(S \backslash\{i\}, \underline{v}_{g}\right)=\left(\phi_{\underline{V}}\right)_{j}(S, g)-\left(\phi_{\underline{V}}\right)_{j}(S \backslash\{i\}, g)$, where the second equality follows from balanced contributions of the Shapley value for the coalitional game $\left(S, \underline{v}_{g}\right)$ and players $i, j \in S$.

Uniqueness. Let $\varphi$ be a valuation function that satisfies the six properties in the statement of the theorem. We start by proving that $\sum_{i \in S} \varphi_{i}(S, g)=$ $\underline{v}_{g}(S)$ for all $S \subset N$. To do so, we consider the function $\Phi$ that associates with every strategic game $g \in G^{N}$ a coalitional game $(N, \Phi(g))$ defined by $\Phi(g)(S)=\sum_{i \in S} \varphi_{i}(S, g)$ for all $S \subset N$. Then individual objectivity, monotonicity, irrelevance of strongly dominated actions, irrelevance of weakly dominated threats and merge invariance of $\varphi$ imply that $\Phi$ satisfies the appropriate equivalents of these five properties for methods of associating a coalitional game with each strategic game. Carpente et al. (2003) proved that the lower value method, which associates with each strategic game $g \in G^{N}$ the coalitional game $\left(N, \underline{v}_{g}\right)$, is the unique method of associating a coalitional game with each strategic game that satisfies these five properties. Hence, we know $\Phi(g)(S)=\underline{v}_{g}(S)$ for all $g \in G^{N}$ and for all 
$S \subset N$, which of course proves that $\sum_{i \in S} \varphi_{i}(S, g)=\underline{v}_{g}(S)$ for all $g \in G^{N}$ and for all $S \subset N$.

Suppose that $\varphi^{1}$ and $\varphi^{2}$ are two valuation functions satisfying the six properties in the statement of the theorem. Let $g \in G^{N}$. We prove that $\varphi^{1}(S, g)=\varphi^{2}(S, g)$ for all $S \subset N$ by induction to the size of $S$.

If $S=\{i\}$, then $\varphi_{i}^{1}(S, g)=\sum_{j \in S} \varphi_{j}^{1}(S, g)=\underline{v}_{g}(S)=\sum_{j \in S} \varphi_{j}^{2}(S, g)=$ $\varphi_{i}^{2}(S, g)$. Now, suppose we have proved that $\varphi^{1}(S, g)=\varphi^{2}(S, g)$ for all $S \subset$ $N$ with $|S| \leq t$, where $1 \leq t<n$. Let $S \subset N$ with $|S|=t+1$. Using balanced contributions of $\varphi^{l}, l=1,2$, we derive that for all $i, j \in S, i \neq j, \varphi_{j}^{1}(S, g)-$ $\varphi_{i}^{1}(S, g)=\varphi_{j}^{1}(S \backslash\{i\}, g)-\varphi_{i}^{1}(S \backslash\{j\}, g)=\varphi_{j}^{2}(S \backslash\{i\}, g)-\varphi_{i}^{2}(S \backslash\{j\}, g)=$ $\varphi_{j}^{2}(S, g)-\varphi_{i}^{2}(S, g)$, where the second equality uses the induction hypothesis. Together with $\underline{v}_{g}(S)=\sum_{i \in S} \varphi_{i}^{1}(S, g)=\sum_{i \in S} \varphi_{i}^{2}(S, g)$, this implies that $\varphi_{i}^{1}(S, g)=\varphi_{i}^{2}(S, g)$ for all $i \in S$. This finishes the proof of the theorem.

\section{Concluding remarks}

In Section 2 we defined and axiomatically characterized the Shapley valuation function, which associates with each strategic game and cooperating coalition of players a payoff vector that provides a valuation for each of the members of the coalition. The Shapley valuation function is defined using the lower-value based method to associate a coalitional game with each strategic game that was introduced in Carpente et al. (2003). As argued in that paper, the use of the lower value is appropriate in settings in which mixing coordinated actions is not possible or reasonable. In situations where the use of such strategies is possible, however, the value based method introduced in Von Neumann and Morgenstern (1944) can be used to construct a valuation function $\phi_{V}$ by considering Shapley values of coalitional games $\left(S, v_{g}\right)$ that are defined using this value based method. Given a strategic game $g \in G^{N}$, this method defines the worth $v_{g}(S)$ of a coalition of players $S \subset N$ as the value of the mixed extension of the two-person zero-sum game $g_{S}$ between coalitions $S$ and $N \backslash S$. The axiomatic characterization of this method in Carpente et al. (2003) can be used to find an axiomatic 
characterization of the valuation function $\phi_{V}$. In addition to some of the properties that we have already encountered, this axiomatization uses the following two properties.

Irrelevance of dominated actions. In a game $g \in G^{N}$, an action $x_{i} \in X_{i}$ of player $i \in N$ is dominated if there exists a convex combination $y$ of the other actions of player $i$, with the property that $u_{i}\left(y, x_{N \backslash i}\right) \geq u_{i}\left(x_{i}, x_{N \backslash i}\right)$ for all $x_{N \backslash i} \in X_{N \backslash i}{ }^{1}$ For all $g \in G^{N}$ and $i \in N$, if action $x_{i} \in X_{i}$ is dominated, then $\varphi_{i}(\{i\}, g)=\varphi_{i}\left(\{i\}, g^{\prime}\right)$, where $g^{\prime} \in G^{N}$ is the game obtained from $g$ by deleting action $x_{i}$.

Irrelevance of dominated threats. In a game $g \in G^{N}$, an action $x_{j} \in X_{j}$ of a player $j \in N$ is a dominated threat to player $i \in N, i \neq j$, if there exists a convex combination $y$ of the other actions of player $j$ with the property that $u_{i}\left(y, x_{N \backslash j}\right) \leq u_{i}\left(x_{j}, x_{N \backslash j}\right)$ for all $x_{N \backslash j} \in X_{N \backslash j}$. For all $g \in G^{N}$ and players $i, j \in N, i \neq j$, if action $x_{j} \in X_{j}$ is a dominated threat to player $i$, then $\varphi_{i}(\{i\}, g)=\varphi_{i}\left(\{i\}, g^{\prime}\right)$, where $g^{\prime} \in G^{N}$ is the game obtained from $g$ by deleting action $x_{j}$.

Irrelevance of dominated actions states that the valuation for a player of forming a singleton coalition does not change if in the strategic game he loses the ability to use an action that is weakly worse for him than a mix of his other actions, no matter what actions the other players choose. Note that irrelevance of dominated actions is a stronger property than irrelevance of strongly dominated actions. Irrelevance of dominated threats has a similar interpretation to irrelevance of weakly dominated threats but is weaker than that property as every threat that is dominated is also weakly dominated.

Theorem 2 The valuation function $\phi_{V}$ is the unique valuation function satisfying individual objectivity, monotonicity, irrelevance of dominated actions, irrelevance of dominated threats, merge invariance, and balanced contributions.

\footnotetext{
${ }^{1} u_{i}\left(y, x_{N \backslash i}\right):=\sum_{\hat{x}_{i} \in X_{i}} y\left(\hat{x}_{i}\right) u_{i}\left(\hat{x}_{i}, x_{N \backslash i}\right)$, where $y=\sum_{\hat{x}_{i} \in X_{i}} y\left(\hat{x}_{i}\right) \hat{x}_{i}$. Note that $y\left(x_{i}\right)=0, y\left(\hat{x}_{i}\right) \geq 0$, for all $\hat{x}_{i} \in X_{i}$, and $\sum_{\hat{x}_{i} \in X_{i}} y\left(\hat{x}_{i}\right)=1$. Observe that $y$ is simply a mixed strategy of player $i$.
} 
Theorem 2 can be proved in a manner similar to the proof of Theorem 1 by using Carpente et al.'s axiomatic characterization of Von Neumann and Morgenstern's value based method.

We conclude this note by pointing out that in the axiomatic characterizations in Theorems 1 and 2 monotonicity can be replaced by two properties called elimination of own actions and elimination of others' actions. These two properties are inspired by the properties irrelevance of (strongly) dominated actions and irrelevance of (weakly) dominated threats and address the elimination of arbitrary actions, dominated or not. Elimination of own actions states that the elimination of an (arbitrary) action of player $i$ does not increase this player's valuation of forming a singleton coalition, and elimination of others' actions states that the valuation for a player $i$ of forming a singleton coalition does not decrease when an (arbitrary) action of another player $j$ is eliminated. Hence, these properties highlight a form of monotonicity with respect to the elimination of actions. It is shown in Carpente et al. (2003) that these properties can replace monotonicity in the axiomatic characterizations of both the value based and the lower-value based methods to associate a coalitional game with each strategic game and this result can be adapted to the valuation function setting of this note. These properties have also been used in Norde and Voorneveld (2003) to characterize the value of the mixed extension of a matrix game.

\section{References}

Carpente L, Casas-Méndez B, García-Jurado I, Van den Nouweland A (2003) Values for strategic games in which players cooperate. To appear in International Journal of Game Theory.

Myerson RB (1980) Conference structures and fair allocation rules. International Journal of Game Theory 9:169-182.

Norde H, Voorneveld M (2003) Axiomatizations of the value of matrix games. CentER Discussion Paper 2003-17. Tilburg University.

Shapley LS (1953) A value for n-person games. Annals of Mathematics 
Studies 28:307-318.

Von Neumann J, Morgenstern O (1944) Theory of Games and Economic Behavior. Princeton University Press. 